\title{
Editorial 1st international Pichia anomala mini-symposium
}

\author{
Heide-Marie Daniel • Volkmar Passoth • \\ Graeme Walker
}

Received: 27 October 2010/Accepted: 4 December 2010/Published online: 29 December 2010

(C) Springer Science+Business Media B.V. 2010

Yeast species, albeit morphologically relatively uniform, exhibit a wide biodiversity in terms of cell physiology and adaptation to specific habitats. Yeasts are also widely exploited in traditional and modern biotechnologies, whilst some species act as food spoilers and opportunistic human pathogens. Saccharomyces cerevisiae represents the most explored and exploited yeast, but numerous non-Saccharomyces species are now the focus of dedicated yeast researchers. These include Pichia anomala, the subject of this Special Issue, which documents the converging interests of researchers working on biocontrol, ecology, enzyme production and formulation, food and feed microbiology and preservation, genetics, infectious diseases, legal regulations, phylogeny and taxonomy, and physiology. The idea to

H.-M. Daniel ( $₫)$

Mycothèque de l'Université catholique de Louvain (MUCL), Member of the Belgian Coordinated Collection of Microorganisms (BCCM), Earth and Life Institut, Applied Microbiology, Mycology, Université catholique de Louvain, Croix du Sud 3, bte 6, 1348 Louvain-laNeuve, Belgium

e-mail: heide-marie.daniel@uclouvain.be

V. Passoth

Department of Microbiology, Swedish University of Agricultural Sciences, Box 7025, 75007 Uppsala, Sweden

G. Walker

Yeast Research Group, School of Contemporary Sciences, University of Abertay Dundee, Bell Street, Dundee, DD1 1HG Scotland, UK meet and exchange interdisciplinary knowledge on this singular yeast species was promoted by Professor Johan Schnürer during and after the 12th International Congress on Yeasts, 2008 in Kiev. The resulting meeting, the 1st International Pichia anomala mini-Symposium, was hosted by the Swedish University of Agricultural Sciences and held in Uppsala from 10th to 12th February 2010, sponsored by the MicroDrivE (http://microdrive.phosdev.se/) and DOM-(http://www.mistra.org/dom) research programs. During this meeting the wish was expressed to communicate the diverse knowledge on $P$. anomala in a compendium, which is now represented by this Special Issue. Most of the fourteen meeting presentations and one independently submitted work on the action of an exo- $\beta$-1,3-glucanase isolated from P. anomala on two Penicillium species (Izgu et al.) are presented herein mainly in the form of brief review-like papers. These contributions direct the interested reader to the more detailed original sources of research on this fascinating yeast.

Uppsala should not only be known as the final workplace of Linnaeus, founder of binominal nomenclature, but also as a key location of research on $P$. anomala in the biopreservation of moist grain since the early 1980s. Here, the yeast was found to dominate large-scale experimental grain silos while inhibiting mould growth. This observation led to research into the mechanisms and application of this phenomenon directed by Schnürer and colleagues. The resulting contributions include an introduction 
and overview of early $P$. anomala biocontrol research (Schnürer and Jonsson), an evaluation of the safety regulations applied to yeasts in the food- and feed chains (Sundh and Melin), an account of the formulation and stabilization of $P$. anomala in view of industrial application (presented by Håkansson), the aspects of avoiding high energy costs by using $P$. anomala for preserving moist grain for animal feed (Olstorpe) and an review and outlook into the future with the sequencing of the $P$. anomala genome on its way at the Joint Genome Institute and the planned exploration of its transcriptome (Passoth).

Other interesting industrial, environmental and medical aspects of $P$. anomala research were also presented at the Symposium and in this Special Issue. The production of phytase by this yeast and its application as a feed supplement was presented by Satyanarayana. Phytases also have beneficial impacts on human nutrition using cereal products. The finding of large and apparently homogeneous populations of $P$. anomala in sourdough needs further study to affirm the role this yeast may play in mixed microbial systems containing different yeasts and lactic acid bacteria (Daniel et al.). Studies of another man-made complex ecosystem, that of malting, has revealed potentially advantageous roles of $P$. anomala by inhibiting production of hydrophobin, a protein from contaminating filamentous fungi that causes severe quality defects in beer, such as gushing, unwanted extensive foaming (Laitila et al.). Important losses of fruits such as apples are still encountered during storage due to microbial (mainly fungal) contamination. The application of $P$. anomala as a biological control agent has great potential as an alternative to the current use of synthetic fungicides. Research lead by Jijakli has pointed towards the specific role of a gene encoding a $\beta$-1,3-glucanase for the efficiency of biocontrol of post-harvest fruits. Detailed studies of immune reactions by antibodies to the $\beta$-glucan receptors of $P$. anomala killer protein may lead to a new generation of anti-infective agents. For example, a peptide reflecting the active region of a $P$. anomala killer protein has been shown by Polonelli et al. to be active against major fungal, bacterial, parasitic and viral pathogens. The isolation of $P$. anomala from the midgut and reproductive organs of the main Asian malaria vector (Anopheles stephensi), communicated by Mosca, may eventually lead to the yeast being applied in "symbiotic control" of mosquito-borne diseases. When compared to $S$. cerevisiae, $P$. anomala shows a number of notable differences. These were discussed by Walker and include diverse habitats; nutritional diversity with regard to $\mathrm{C}, \mathrm{N}$ and $\mathrm{P}$ sources; pronounced stress tolerance; a missing Crabtree effect leading to respiratory metabolism under high glucose concentrations in aerobic conditions; different chromosomally encoded killer proteins; and a different ester synthesis pathway. Regarding $P$. anomala esters, ethyl acetate is the predominant volatile ester and is thought to be instrumental combating fungal growth.

The editors have chosen to use the name $P$. anomala for this editorial in adherence to the symposium title. The species $P$. anomala has been assigned to different genera by reclassifications based on ascospore morphology and physiological growth characteristics. It is best known as Hansenula anomala (E.C. Hansen) Syd. and P. Syd. and Pichia anomala (E.C. Hansen) Kurtzman and has been renamed recently as Wickerhamomyces anomalus (E.C. Hansen) Kurtzman, Robnett and BasehoarPowers (see contribution by Kurtzman). Originally described as Saccharomyces anomalus by Hansen (1889), this species is a testament to the constant refinements of taxonomy from the very early stages of yeast classification, when only few genera were recognized and available to accommodate the already growing number of species. The increasing understanding of yeast biodiversity is currently acknowledged by the description of numerous new genera that will help future generations to communicate more efficiently the specific properties of particular groups of yeasts. To avoid uncertainty in the process of re-naming, the application of nomenclatural rules and the principles of molecular systematics are of fundamental importance. At the Uppsala Symposium, Naumov and Naumova mentioned a possible priority of the name Hansenula. They also presented data on the genetics of $P$. anomala including strain polymorphisms regarding sporulation level, mating type activities, ascospore viability, ploidy and karyotype. Despite difficulties they succeeded in creating fertile haploid genetic lines, the essential condition for genetic manipulation. Naumov and Naumova consider $P$. anomala as homothallic yeast showing delayed self-diploidization resulting in a tetraploid status for many natural strains. We are looking forward to presenting their viewpoint on the 
taxonomy of $P$. anomala in a later issue of the Antonie van Leeuwenhoek Journal of Microbiology.

It is our hope that the contributions presented in this Special Issue on P. anomala will be of interest to the yeast community and to microbiologists in general as it raises important issues linked to natural resources, improvement of food quality, and the prevention and treatment of diseases.

Heide-Marie Daniel, Volkmar Passoth and Graeme Walker (Guest Editor). 